\title{
Searching for Sterile Neutrinos and CP Violation: The IsoDAR and DAE $\delta$ ALUS Experiments
}

\author{
M. H. Shaevitz* \\ Columbia University; New York, NY 10027 \\ E-mail: shaevitz@nevis.columbia.edu \\ for the IsoDAR/DAESALUS Collaboration
}

The IsoDAR experiment uses a novel isotope decay-at-rest (DAR) source of electron antineutrinos using protons from a $60 \mathrm{MeV}$ cyclotron. Paired with a large neutrino detector (such as KamLAND, WATCHMAN, or JUNO), the experiment can observe hundreds of thousands of inverse beta-decay events and do a decisive test of the current hints for sterile neutrino. DAE $\delta$ ALUS is a phased program leading to a high-sensitivity search for $\mathrm{CP}$ violation. The experiment uses a set of high-intensity $800 \mathrm{MeV}$ cyclotrons to produce pion DAR neutrino sources at several locations $(1.5 \mathrm{~km}, 8 \mathrm{~km}$, and $20 \mathrm{~km})$ going to a single ultra-large, underground detector with free protons such as Hyper-K. The DAE $\delta$ ALUS experiment will provide a high-statistics antineutrino data set with no matter effects that can be combined with long-baseline data sets to provide enhanced sensitivity to $\mathrm{CP}$ violation and matter effects.

XVI International Workshop on Neutrino Telescopes,

2-6 March 2015

Palazzo Franchetti âĂŞ Istituto Veneto, Venice, Italy

\footnotetext{
*Speaker.
} 


\section{Introduction}

DAE $\delta$ ALUS (Decay-At-rest Experiment for $\delta_{C P}$ studies At the Laboratory for Underground Science) is a phased R\&D program leading to a high-sensitivity search for $C P$-violation $[1,2]$. This is a unique, cyclotron-driven $\bar{v}_{\mu} \rightarrow \bar{v}_{e}$ search for a non-zero $C P$ violation parameter, $\delta_{C P}$, in the three-neutrino mixing matrix. DAE $\delta$ ALUS, when combined with Hyper K (with-JPARC beam), can achieve an uncertainty of 4 to 12 degrees on $\delta_{C P}$-well beyond the sensitivity of LBNE or HyperK alone. The system consists of a two-cyclotron design. The smaller injector cyclotron, which will be developed first, also can be used as a driver to provide a high intensity, very pure source of $\bar{v}_{e}$ 's. The source can be paired with a large detector such as KamLAND to do a disappearance search for oscillations to sterile neutrinos at a sensitivity five time better than other current proposals. This isotope decay-at-rest experiment is referred to as IsoDAR. DAE $\delta$ ALUS and IsoDAR are two examples of applications of these new type of high-intensity cyclotrons. However, one can envision uses beyond these within neutrino physics, including cross sections and other beyond the standard model searches. These cyclotrons are also valuable commercially, which is why DAE $\delta$ ALUS has a strong industry-university collaboration for the R\&D program.

\section{The IsoDAR Experiment}

Searches for light sterile neutrinos with mass $\sim 1 \mathrm{eV}$ are motivated by observed anomalies in several experiments. Intriguingly, these results come from a wide range of experiments covering neutrinos, anti-neutrinos, different flavors, and different energies. Short baseline accelerator neutrino oscillation experiments [3,4], short baseline reactor experiments [5, 6], and even the radioactive source experiments, which were originally intended as calibrations for the chemical solar neutrino experiments $[7,8]$, have all observed anomalies that can be interpreted as due to one or more sterile neutrinos.

To understand these anomalies in terms of the $v \mathrm{SM}$ for neutrino oscillations, the observations must be compared to the data from the large range of experiments with null results $[9,10,11,12]$, and then to a model. These "global fits" are most often to models with one or more sterile neutrinos added to the oscillation probability calculation [13]. The extended models are referred to as " $3+1$ ", " $3+2$ ", or " $3+3$ " neutrino models depending on the number of additional sterile neutrinos. The global fits tend to prefer $3+2$ and $3+3$ models with $C P$ violation; $3+1$ models are very hard to reconcile between the experiments with signals and those with null results [14].

The diversity of experiments showing these anomalous results has motivated a number of short baseline proposals to address them. Suggestions range from repeating the source experiments, to specially designed reactor antineutrino experiments, to accelerator-based ones. Many of these proposals, however, do not have sufficient sensitivity to make a definitive $>5 \sigma$ statement about the existence of sterile neutrinos in all of the relevant parameter space. In addition, the "smoking gun" proof for oscillations would be observation of oscillatory behavior of the neutrino detection rate with distance and energy.

The IsoDAR experiment [15] would use a DAE $\delta$ ALUS style injector cyclotron to generate an isotope DAR neutrino source. The IsoDAR experimental program has the goal of performing a number of unique searches associated with neutrino oscillations and non-standard neutrino 
interactions. The experiment is being designed to definitively address these physics topics using a well-understood, high-intensity ${ }^{8} \mathrm{Li} \beta$-decay-at-rest antineutrino source coupled with a massive detector such as KamLAND [16] or WATCHMAN [17] that has good inverse-beta-decay identification capabilities with high efficiency. Such a combination would, for example, enable a definitive search for sterile neutrinos by observing a deficit of antineutrinos as a function of the distance $L$ and antineutrino energy $E$ across the detector.

The high statistics data sample will allow excellent sensitivity to these new physics signatures and also provide the ability to study any signals that are detected. Accurately mapping out the oscillation wave within the detector will not only conclusively test if a signal is associated with neutrino oscillation rather than background but also give new handles for studying any observed new physics signals. For example, the oscillation wave can be used to determine the number of extra sterile neutrino flavors by differentiating the oscillatory behavior of a $(3+1)$ versus a $(3+2)$ oscillation model. The high intensity also will allow the study of antineutrino-electron scattering. This is a very clean and well-understood interaction that can be used to search for indications of non-standard neutrino interactions proposed in many extensions of the standard model.

The IsoDAR neutrino source [15] consists of an accelerator producing $60 \mathrm{MeV}$ protons that impinge on a ${ }^{9} \mathrm{Be}$ target, producing neutrons. The protons enter a surrounding $\geq 99.99 \%$ isotopically pure ${ }^{7} \mathrm{Li}$ sleeve, where neutron capture results in ${ }^{8} \mathrm{Li}$; this isotope undergoes $\beta$ decay at rest to produce an isotropic $\bar{v}_{e}$ flux with an average energy of $\sim 6.4 \mathrm{MeV}$ and an endpoint of $\sim 13 \mathrm{MeV}$. The $\bar{v}_{e}$ will interact in a scintillator or water detector via inverse beta decay (IBD), $\bar{v}_{e}+p \rightarrow e^{+}+n$, which is easily tagged through prompt-light plus neutron-capture coincidence. When paired with a kiloton size detector such as KamLAND, the experiment could observe $8.2 \times 10^{5}$ reconstructed IBD events in five years. With this data set, IsoDAR could decisively test sterile neutrino oscillation models, allow precision measurement of $\bar{v}_{e}-e$ scattering, and search for production and decay of exotic particles.

As an example, the proposed IsoDAR target could be placed adjacent to the KamLAND detector [16]. The antineutrinos propagate $9.5 \mathrm{~m}$ through a combination of rock, outer muon veto, and buffer liquid to the active scintillator volume of KamLAND. The scintillator is contained in a nylon balloon $6.5 \mathrm{~m}$ in radius bringing the total distance from target to detector center to $16.1 \mathrm{~m}$. The antineutrinos are then detected via the IBD interaction. This interaction has a well known cross section with an uncertainty of $0.2 \%$, and creates a distinctive coincidence signal between a prompt positron signal, $E_{v i s}=E_{\bar{v}_{e}}-0.78 \mathrm{MeV}$, and a delayed neutron capture giving a $2.2 \mathrm{MeV}$ gamma ray within $\sim 200 \mu \mathrm{s}$.

KamLAND was designed to efficiently detect IBD. A standard analysis has a $92 \%$ efficiency for identifying IBD events. In IsoDAR's nominal 5 year run, $8.2 \times 10^{5}$ IBD events are expected. The largest background comes from the 100 reactor antineutrino IBD events detected by KamLAND per year depending on the reactor operations. The sterile neutrino analysis uses an energy threshold of $3 \mathrm{MeV}$. Due to the effective background rejection efficiency provided by the IBD delayed coincidence signal, this threshold enables use of the full KamLAND fiducial volume, $\mathrm{R}<6.5 \mathrm{~m}$ and 897 metric tons, with negligible backgrounds from sources other than from the aforementioned reactor antineutrinos.

The sterile neutrino analysis makes use of neutrino oscillations's $L / E$ signature. Therefore, the energy and vertex resolutions are essential in determining sensitivity. The KamLAND de- 

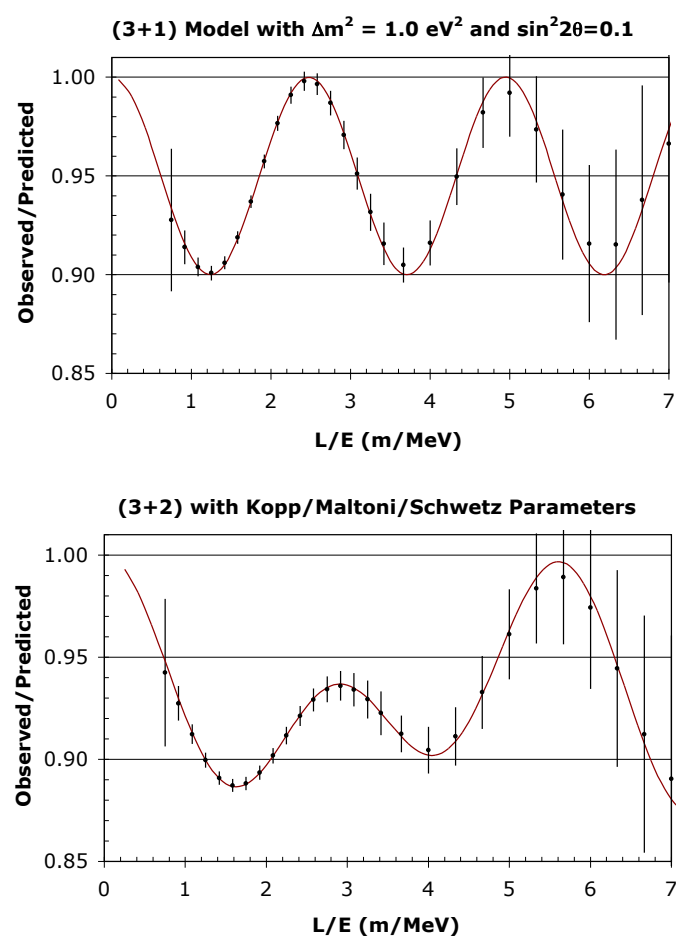

Figure 1: The $L / E$ dependence of sample IsoDAR@KamLAND data sets for 5 years of running for $3+1$ (top) and $3+2$ (bottom) oscillation scenarios. The solid curve is the oscillation probability with no smearing in the reconstructed position and energy and the data points with error bars are from simulated events including smearing. The $3+2$ example (bottom) represents oscillations with the global best fit 3+2 parameters from Ref. [18].

tector has a vertex reconstruction resolution of $12 \mathrm{~cm} / \sqrt{E(\mathrm{MeV})}$ and an energy resolution of $6.4 \% / \sqrt{E(\mathrm{MeV})}$. Sample data sets for reasonable $3+1$ and $3+2$ sterile models are shown in Figure 1. In most currently favored oscillation scenarios, the $L / E$ signal would be observable in IsoDAR. Furthermore, separation of the various $3+\mathrm{N}$ models may be possible as exemplified by Figure 1 (bottom).

To understand the IsoDAR sensitivity relative to current allowed regions, the $5 \sigma$ sensitivity is compared to the allowed global fit [19] and "Reactor Anomaly" regions in Figure 2. With three years of data, IsoDAR@KamLAND would cover the full allowed regions associated with the global fits and the "Reactor Anomaly" up to the $10 \mathrm{eV}^{2}$. Also IsoDAR@WATCHMAN with a pure water detector would cover this global fit region with 3 years of data.

Recently there have been investigations of reactor neutrino experiments directed towards measuring the mass hierarchy associated with neutrino oscillation. One example is the JUNO experiment [20] that would use a large $20 \mathrm{kton}, 34.5 \mathrm{~m}$ diameter liquid scintillator detector placed $50 \mathrm{~km}$ from the Yangjiang and Taishan $36 \mathrm{GW}$ reactor complex. The JUNO detector will have excellent energy and vertex resolution and when coupled with an IsoDAR antineutrino source at $25 \mathrm{~m}$ from the detector center would have unprecedented sensitivity to oscillations to sterile neutrinos [21]. As shown in Figure 3, the IsoDAR at JUNO experiment with 5 years of data could completely cover not only the current electron disappearance signal regions but also the full electron neutrino appearance region associated with the MiniBooNE and LSND signals. 


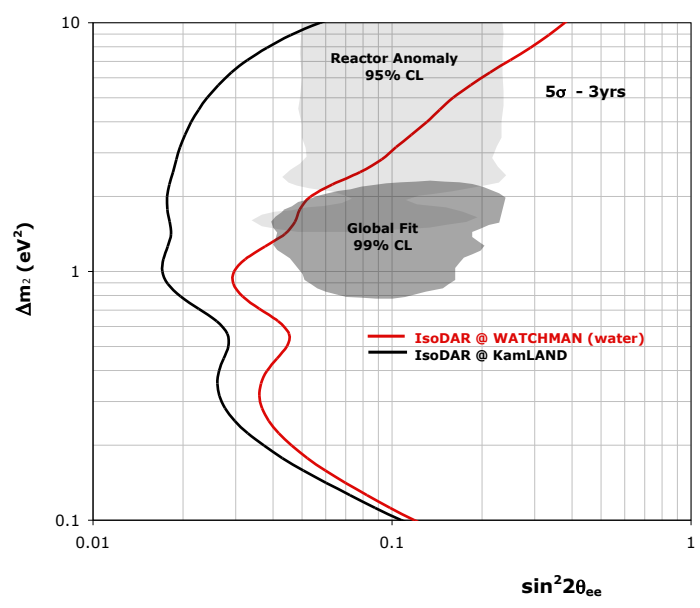

Figure 2: Sensitivity of IsoDAR at $5 \sigma$ for a nominal 3 year run with either the KamLAND (scintillator) or WATCHMAN (pure water) detector compared to the allowed global fit [19] and "Reactor Anomaly" regions.

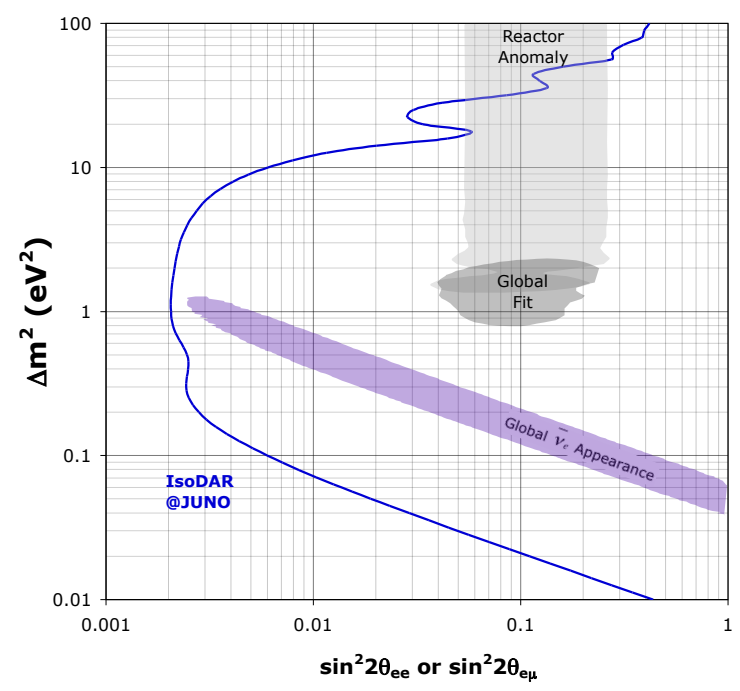

Figure 3: Allowed regions versus the sensitivity for the IsoDAR at JUNO experiment. The blue curve indicates the $\Delta m^{2}$ vs. $\sin ^{2} 2 \theta_{e e}$ boundaries where the null oscillation hypothesis can be excluded at $5 \sigma$ for a five year data run of IsoDAR at JUNO. The purple area is the $99 \% \mathrm{CL}$ for a combined fit to all $\bar{v}_{e}$ appearance data[14], plotted as $\Delta m^{2}$ vs. $\sin ^{2} 2 \theta_{e \mu}$. Plot is adapted from Ref. [21],

\section{The DAE $\delta$ ALUS Experiment}

$C P$ violation can occur in neutrino oscillations if there is a complex phase, $\delta_{C P}$, in the $3 \times$ 3 neutrino mixing matrix between the neutrino flavor and mass eigenstates. Observation of $\mathrm{CP}$ violation in the light neutrino sector would be a first hint of a possible "lepto-genesis" process in the early universe where GUT-scale Majorana neutrinos can have $C P$ violating decays that lead to the matter-antimatter asymmetry that we now observe.

The parameter $\delta_{C P}$ is accessible through the muon-to-electron neutrino flavor oscillation prob- 
ability. For oscillations in a vacuum, the probability is given by:

$$
\begin{aligned}
& P_{\mu \rightarrow e}=\sin ^{2} \theta_{23} \sin ^{2} 2 \theta_{13} \sin ^{2} \Delta_{31} \\
& \mp \sin \delta \sin 2 \theta_{13} \sin 2 \theta_{23} \sin 2 \theta_{12} \sin ^{2} \Delta_{31} \sin \Delta_{21} \\
& +\cos \delta \sin 2 \theta_{13} \sin 2 \theta_{23} \sin 2 \theta_{12} \sin \Delta_{31} \cos \Delta_{31} \sin \Delta_{21} \\
& +\cos ^{2} \theta_{23} \sin ^{2} 2 \theta_{12} \sin ^{2} \Delta_{21}
\end{aligned}
$$

where $\Delta_{i j}=\Delta m_{i j}^{2} L / 4 E_{v}$. In the second term, the $-(+)$ refers to neutrino (antineutrino) oscillations. The fact that $\theta_{13}$ is now known to be fairly large makes the search for $C P$ violation viable and a key next step in particle physics.

For long baseline experiments, searches for $C P$ violation rely on comparing neutrino and antineutrino oscillation probabilities, thus, exploiting the above change of sign in order to isolate $\delta_{C P}$. This type of measurement is complicated by matter effects, in which the forward scattering amplitude for neutrinos and antineutrinos differs due to the presence of electrons, rather than positrons, in matter. The matter effects result in a modification of Eq. 3.1 when $L$ is large. Short-baseline experiments, such as DAE $\delta$ ALUS and moderate baseline experiments such as T2K at $L=295$ $\mathrm{km}$ suffer negligible matter effects. On the other hand, long baseline experiments such as NOvA and LBNE have significant matter effects. The terms that are modified by the matter effects also depend on $\operatorname{sign}\left(\Delta m_{31}^{2}\right)$, making the corrections dependent on knowing this sign, commonly called the "mass hierarchy."

In addition for long baseline accelerator oscillation experiments, gathering a sufficient antineutrino data set is difficult due to the reduced negative pion production rate by accelerator protons and by the reduced interaction cross section of antineutrinos. Also, the antineutrino data set will have significant neutrino contamination since it is difficult to sweep out the unwanted positive pions. Finally, these accelerator neutrino beams typically have high energy tails in the neutrino distribution that contributes little to oscillation parameter sensitivity but increases the neutral current backgrounds and other backgrounds to the measurement.

In contrast, the DAE $\delta$ ALUS experiment will be a search only in the antineutrino mode $\bar{v}_{\mu} \rightarrow$ $\bar{v}_{e}$ with no matter effects, and with reduced backgrounds and systematic uncertainties, plus a unique experimental layout in which several low-cost neutrino sources are at different distances from one large detector. With an antineutrino-only beam, the oscillation probability is given by Eq. 3.1, and the sensitivity to $C P$ violation comes about through the interference between $\Delta_{12}$ and $\Delta_{13}$ transitions, which have a distinctive $L$ dependence.

Specifically, DAE $\delta$ ALUS will search for $\bar{v}_{\mu} \rightarrow \bar{v}_{e}$ oscillations using neutrinos from three stopped-pion DAR sources driven by $800 \mathrm{MeV}$ cyclotrons, which interact in a single large 200 to 500 kton Gd-doped water Cherenkov or a large 50 kton liquid scintillator detector. Pion decayat-rest (PDAR) neutrino sources, produced through $\pi \rightarrow \mu \rightarrow \nu$, offer a precision alternative to pion decay-in-flight neutrino beams. PDAR sources have well defined flavor content and energy distributions and produce equal amounts of $v_{\mu}, \bar{v}_{\mu}$, and $v_{e}$ isotropically in the energy range from a few MeV to $52.8 \mathrm{MeV}$. The PDAR is, thus, ideal for observing $\bar{v}_{\mu} \rightarrow \bar{v}_{e}$ oscillations.

For DAE $\delta$ ALUS, the pion production will accomplished using $800 \mathrm{MeV}$ protons from highpower cyclotrons ( $\sim 10 \mathrm{ma}$ ) impinging on a light target to produce a high rate of pions through the $\Delta$-resonance. The target must be surrounded by heavy material to stop the outgoing pions before 
DIF. In this case, the neutrinos originate primarily from $\pi^{+}$or $\mu^{+}$decay. The negatively charged pions and muons stop and capture on nuclei before they can decay to produce neutrinos. The production of kaons or heavier mesons, which could produce unwanted backgrounds, is negligible if the primary proton energy is below about $1 \mathrm{GeV}$. The $\bar{v}_{e}$ flux can be maintained at the level $\sim 5 \times 10^{-4}$ of the $\bar{v}_{\mu}$ flux in the $20<E_{v}<52.8 \mathrm{MeV}$ energy range. As a result, the source is ideal for observing $\bar{v}_{\mu} \rightarrow \bar{v}_{e}$ oscillations [2].

In DAE $\delta$ ALUS, the detection of the electron antineutrinos from oscillations would be done using the IBD process where the outgoing positron is once again required to have a delayed coincidence with a neutron capture on Gd for the water detector or on hydrogen for the scintillator detector. This process has a high cross section at $\sim 50 \mathrm{MeV}$, but requires either Gd doping for a water detector or a scintillator detector to detect the outgoing neutron and separate the IBD events from the preponderance of charged-current $v_{e}$ events.

The accelerator DAR neutrino sources will be positioned at 1.5, 8, and $20 \mathrm{~km}$ from the large detector and are all above ground to reduce the installation and running complexity. Each accelerator provides different physics data for the $C P$ violation search. The $1.5-\mathrm{km}$ accelerator allows measurement of the beam-on backgrounds and the normalization. The $8 \mathrm{~km}$ site is at an oscillation wavelength of about $\pi / 4$ at $50 \mathrm{MeV}$ and the $20 \mathrm{~km}$ site is at oscillation maximum for this energy. Each site will be run for $20 \%$ of the time so that the events from a given source distance can be identified by their time-stamp with respect to this running cycle. This will leave $40 \%$ of the time for beam-off running to measure the non-beam backgrounds and provide other physics data. The baseline plan is for a ten year run with $1 \mathrm{MW}, 2 \mathrm{MW}$, and $5 \mathrm{MW}$ neutrino sources at the $1.5,8$, and $20 \mathrm{~km}$ sites, respectively.

Combining the data from the three accelerators helps to minimize the systematic uncertainties associated with the beam and detector and leads to a highly sensitive search for $C P$ violation. The shape of the DAR flux with energy is known to high precision and is common among the various distances; thus shape comparisons will have small uncertainties. The interaction and detector systematic errors are low since all events are detected in a single detector. The fiducial volume error on the IBD events is also small due to the extreme volume-to-surface-area ratio of the ultra-large detector. Therefore, the main errors for the measurements are related to the statistics of the data and to normalization uncertainties. The normalization uncertainties are dominated by the neutron tagging efficiency, assumed to be $0.5 \%$, and the antineutrino flux uncertainties that are constrained as described next.

The DAE $\delta$ ALUS $C P$ violation analysis follows three steps. First, the absolute normalization of the flux from the near accelerator is measured using the $>21,000$ neutrino-electron scatters from that source in the detector, for which the cross section is known to $1 \%$. The relative flux normalization between the sources is then determined using the comparative rates of charged current $v_{e^{-}}$ oxygen (or $v_{e}$-carbon) interactions in the the detector. Since this is a relative measurement, the cross section uncertainty does not come in but the high statistics is important. Once the normalizations of the accelerators are known, then the IBD data can be fit to extract the $C P$-violating parameter $\delta_{C P}$. The fit needs to include all the above systematic uncertainties along with the physics parameter uncertainties associated with, for example, the knowledge of $\sin ^{2} 2 \theta_{13}$ and $\sin ^{2} \theta_{23}$, which are assumed to be known with an error of \pm 0.005 and \pm 0.01 , respectively.

DAE $\delta$ ALUS must be paired with water or scintillator detectors that have free proton targets. 
The sensitivity studies shown here include the 35 kton LBNE liquid argon detector with the 850 kW FNAL beam [22] and the $560 \mathrm{kton}$ Gd-doped Hyper-K water detector with the $750 \mathrm{~kW}$ JPARC beam [23]. In addition, results are presented for DAE $\delta$ ALUS paired with the Gd-doped $560 \mathrm{kt}$ Hyper-K ("DAE $\delta$ ALUS @ Hyper-K"). This combination results in unprecedented sensitivity to $C P$ violation when "DAE $\delta$ ALUS@ Hyper-K" data is combined with data from Hyper-K running with the $750 \mathrm{~kW}$ JPARC beam. ("DAE $\delta$ ALUS/JPARC@Hyper-K"). In this scenario, JPARC provides a pure $v_{\mu}$ flux, rather than running in neutrino and antineutrino mode. This plays to the strength of the JPARC conventional beam, while DAE $\delta$ ALUS provides a high statistics $\bar{v}_{\mu}$ flux with no $v_{\mu}$ contamination.

$C P$ violation sensitivities have been estimated for 10 year data sets for the various configurations using a $\Delta \chi^{2}$ fit with pull parameters for each of the systematic uncertainties. A ten year neutrino-only run of the JPARC@ Hyper-K configuration combined with a ten year DAE $\delta$ ALUS@ Hyper$\mathrm{K}$ exposure is shown in Figure 4. The complementarity of the two experiments is clear and leads to a very precise sensitivity for $C P$ violation with uncertainties estimated to be around $5^{\circ}$.

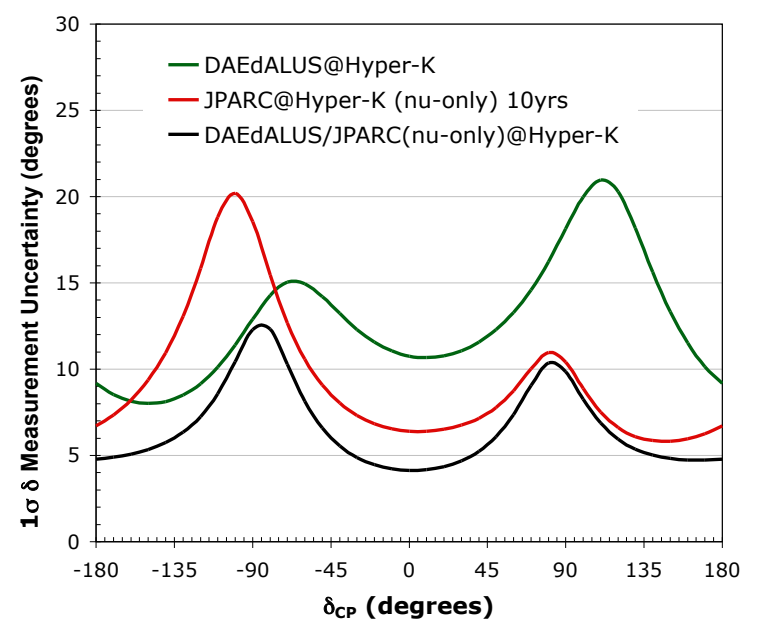

Figure 4: The $\delta_{C P}$ measurement sensitivities at $1 \sigma$ for the DAE $\delta$ ALUS@Hyper-K, JPARC@Hyper-K $(v-$ only $)$, and DAE $\delta$ ALUS/JPARC $(v-$ only) $@$ Hyper-K combined measurement for 10 year data runs.

Finally, Figure 5 shows the cross comparison of several experimental configurations. From this figure, it is clear that the DAE $\delta$ ALUS/JPARC(nu-only)@Hyper-K configuration has excellent sensitivity to $\delta_{C P}$ with a significantly smaller measurement error as compared to the other scenarios.

\section{Summary}

High-power ( $\sim 1 \mathrm{MW})$ class cyclotrons are becoming a reality. For physics, they can provide high intensity neutrino sources. There are also important industrial interests and broader impacts associated with medical isotope production and other applications such as accelerator driven reactors.

For sterile neutrino searches, cyclotrons can be used to produce large rates of isotopes such as ${ }^{8} \mathrm{Li}$ that beta decay giving very high rates of antineutrinos at around $8 \mathrm{MeV}$. The IsoDAR experiment would combine this type of source with a large kton scale detector to make a definitive search 


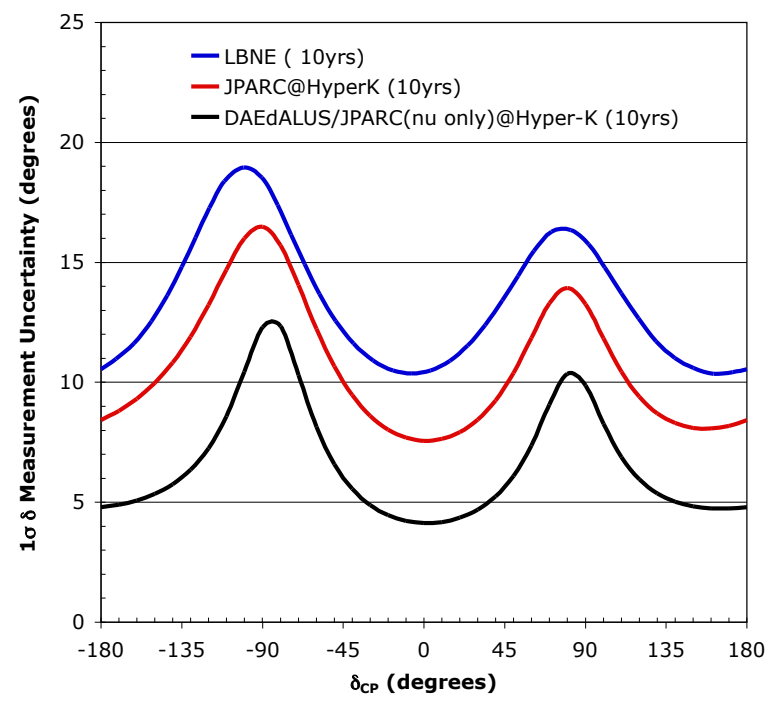

Figure 5: The $\delta_{C P}$ measurement sensitivities at $1 \sigma$ for various configurations: LBNE, JPARC@ Hyper-K, and the DAE $\delta$ ALUS/JPARC( $v$ - only)@Hyper-K combined measurements for 10 year data runs.

for sterile neutrinos using a combined distance and energy analysis. With the good resolutions of the a detector such as KamLAND such an experiment could isolate the oscillatory behavior of the sterile neutrino oscillations and probe the details of a signal if it exists. Establishing the existence of sterile neutrinos with a definitive experiment would be a major result for particle physics.

The DAE $\delta$ ALUS pion decay-at-rest neutrino sources will give another method to probe for CP violation in the neutrino sector. These sources can provide high statistics $\bar{v}_{e}$ appearance data with no matter effects and reduced systematic uncertainty. Combining a DAE $\delta$ ALUS data antineutrino appearance data set with long baseline neutrino appearance data can give significantly enhance $\mathrm{CP}$ violation sensitivity.

\section{References}

[1] A. Adelmann, J. Alonso, W. A. Barletta, J. M. Conrad, M. H. Shaevitz, J. Spitz, M. Toups and L. A. Winslow, Adv. High Energy Phys. 2014, 347097 (2014) [arXiv:1307.6465 [physics.acc-ph]].

[2] J.M. Conrad and M.H. Shaevitz, Phys. Rev. Lett. 104, 141802 (2010).

[3] A. Aguilar et al. [LSND Collaboration], Phys. Rev. D 64, 112007 (2001).

[4] A. A. Aguilar-Arevalo et al. [MiniBooNE Collaboration], Phys. Rev. Lett. 110, 161801 (2013) [arXiv:1207.4809 [hep-ex], arXiv:1303.2588 [hep-ex]].

[5] T. A. Mueller et al., Phys. Rev. C 83, 054615 (2011).

[6] G. Mention, M. Fechner, T. Lasserre, T. A. Mueller, D. Lhuillier, M. Cribier and A. Letourneau, Phys. Rev. D 83, 073006 (2011).

[7] J. N. Abdurashitov et al. [SAGE Collaboration], Phys. Rev. C 80, 015807 (2009).

[8] F. Kaether, W. Hampel, G. Heusser, J. Kiko and T. Kirsten, Phys. Lett. B 685, 47 (2010). 
[9] B. Armbruster et al. [KARMEN Collaboration], Phys. Rev. D 65, 112001 (2002) [hep-ex/0203021].

[10] P. Astier et al. [NOMAD Collaboration], Phys. Lett. B 570, 19 (2003) [hep-ex/0306037].

[11] I.E. Stockdale et al., Phys. Rev. Lett. 52, 1384, (1984).

[12] F. Dydak, G. J. Feldman, C. Guyot, J. P. Merlo, H. J. Meyer, J. Rothberg, J. Steinberger and H. Taureg et al., Phys. Lett. B 134, 281 (1984).

[13] M. Sorel, J. M. Conrad and M. Shaevitz, Phys. Rev. D 70, 073004 (2004) [hep-ph/0305255].

[14] J. M. Conrad, C. M. Ignarra, G. Karagiorgi, M. H. Shaevitz and J. Spitz, Adv. High Energy Phys. 2013, 163897 (2013) [arXiv:1207.4765 [hep-ex]].

[15] A. Bungau et al., Phys. Rev. Lett. 109, 141802 (2012) [arXiv:1205.4419 [hep-ex]].

[16] A. Gando et al. [KamLAND Collaboration], Phys. Rev. D 83, 052002 (2011) [arXiv:1009.4771 [hep-ex]].

[17] M. Askins et al. [WATCHMAN Collaboration], "The Physics and Nuclear Nonproliferation Goals of WATCHMAN: A WAter CHerenkov Monitor for ANtineutrinos,” arXiv:1502.01132 [physics.ins-det]; M. Bergevin et al., (WATCHMAN Collaboration), "A proposal for the design, installation and operation of WATCHMAN: A Water Cherenkov Monitor for Antineutrinos”, www.physics.ucdavis.edu/svoboda/post/WATCHMAN/WATCHMAN_Proposal_Short.pdf

[18] J. Kopp, M. Maltoni and T. Schwetz, Phys. Rev. Lett. 107, 091801 (2011).

[19] C. Giunti and M. Laveder, Phys. Lett. B 706, 200 (2011) [arXiv:1111.1069 [hep-ph]].

[20] M. He [JUNO Collaboration], “Jiangmen Underground Neutrino Observatory,” arXiv:1412.4195 [physics.ins-det].

[21] J. M. Conrad and M. H. Shaevitz, Phys. Rev. D 89, no. 5, 057301 (2014) [arXiv:1310.3857 [hep-ex]].

[22] M. Bishai et al., arXiv:1307.0807 [hep-ex] (2013)

[23] K. Abe, et al. [The Hyper-K Collaboration], arXiv:1109.3262 [hep-ex] (2011). 